\title{
Lyashko-Looijenga morphisms and submaximal factorizations of a Coxeter element
}

\author{
Vivien Ripoll
}

Received: 4 August 2011 / Accepted: 7 February 2012 / Published online: 2 March 2012

(C) Springer Science+Business Media, LLC 2012

\begin{abstract}
When $W$ is a finite reflection group, the noncrossing partition lattice $\mathrm{NC}(W)$ of type $W$ is a rich combinatorial object, extending the notion of noncrossing partitions of an $n$-gon. A formula (for which the only known proofs are case-by-case) expresses the number of multichains of a given length in $\mathrm{NC}(W)$ as a generalized Fuß-Catalan number, depending on the invariant degrees of $W$. We describe how to understand some specifications of this formula in a case-free way, using an interpretation of the chains of $\mathrm{NC}(W)$ as fibers of a Lyashko-Looijenga covering (LL), constructed from the geometry of the discriminant hypersurface of $W$. We study algebraically the map LL, describing the factorizations of its discriminant and its Jacobian. As byproducts, we generalize a formula stated by K. Saito for real reflection groups, and we deduce new enumeration formulas for certain factorizations of a Coxeter element of $W$.
\end{abstract}

Keywords Finite Coxeter group - Complex reflection group - Noncrossing partition lattice $\cdot$ Fuß-Catalan number $\cdot$ Lyashko-Looijenga covering $\cdot$ Coxeter element

\section{Introduction}

Complex reflection groups are a natural generalization of finite real reflection groups (that is, finite Coxeter groups realized in their geometric representation). In this article, we consider a well-generated complex reflection group $W$; the precise definitions will be given in Sect. 2.1.

The noncrossing partition lattice of type $W$, denoted $\mathrm{NC}(W)$, is a particular subset of $W$, endowed with a partial order $\preccurlyeq$ called the absolute order (see definition below). When $W$ is a Coxeter group of type $A, \mathrm{NC}(W)$ is isomorphic to the

V. Ripoll ( $\varangle)$

LaCIM, UQÀM, CP 8888, Succ. Centre-ville Montréal, Montréal, QC, H3C 3P8, Canada

e-mail: vivien.ripoll@lacim.ca 
poset of noncrossing partitions of a set, studied by Kreweras [13]. Throughout the last 15 years, this structure has been generalized to finite Coxeter groups, first (Reiner [18], Bessis [3], Brady-Watt [6]), then to well-generated complex reflection groups (see [4]). It has many applications in the algebraic understanding of the braid group of a reflection group (via the construction of the dual braid monoid, see [3, 4]), and is also studied for itself as a very rich combinatorial object (see Armstrong's memoir [1]).

In order to introduce the structure $\mathrm{NC}(W)$, we need several definitions and notations (which will be detailed in Sect. 2):

- the set $R$ of all reflections of $W$;

- the reflection length (or absolute length) $\ell$ on $W$ : for $w$ in $W, \ell(w)$ is the minimal length of a word on the alphabet $R$ that represents $w$;

- a Coxeter element $c$ in $W$;

- the absolute order $\preccurlyeq$ on $W$, defined as

$$
u \preccurlyeq v \quad \text { if and only if } \ell(u)+\ell\left(u^{-1} v\right)=\ell(v)
$$

The noncrossing partition lattice associated to $(W, c)$ is defined to be the interval below $c$ :

$$
\mathrm{NC}(W)=\{w \in W \mid w \preccurlyeq c\} .
$$

This lattice has a fascinating combinatorics, and one of its most amazing properties concerns its Zeta polynomial (expressing the number of multichains of a given length).

“Chapoton's formula”. Let $W$ be an irreducible, well-generated complex reflection group of rank $n$. Then, for any $p \in \mathbb{N}$, the number of multichains $w_{1} \preccurlyeq \cdots \preccurlyeq w_{p}$ in the poset $\mathrm{NC}(W)$ is equal to

$$
\mathrm{Cat}^{(p)}(W)=\prod_{i=1}^{n} \frac{d_{i}+p h}{d_{i}},
$$

where $d_{1} \leq \ldots \leq d_{n}=h$ are the invariant degrees of $W$ (defined in Sect. 2.1).

The numbers $\mathrm{Cat}^{(p)}(W)$ are called $F u \beta$-Catalan numbers of type $W$ (and Catalan numbers for $p=1$ ). When $W$ is the symmetric group $\mathfrak{S}_{n}$, these are the classical Catalan and Fuß-Catalan numbers $\frac{1}{p n+1}\left(\begin{array}{c}(p+1) n \\ n\end{array}\right)$. Those generalized Fuß-Catalan numbers also appear in other combinatorial objects constructed from the group $W$, for example cluster algebras of finite type introduced in [10] (see Fomin-Reading [9] and the references therein).

In the real case, this formula was first stated by Chapoton in [7, Property 9]. The proof is case-by-case (using the classification of finite Coxeter groups), and it mainly uses results by Athanasiadis and Reiner [2, 18] (see also [16]). The remaining complex cases are checked by Bessis in [4], using results of [5]. There is still no case-free proof of this formula, even for the simplest case $p=1$, which states that the cardi- 
nality of $\mathrm{NC}(W)$ is equal to the generalized Catalan number

$$
\operatorname{Cat}(W)=\prod_{i=1}^{n} \frac{d_{i}+h}{d_{i}} .
$$

This very simple formula naturally incites to look for a uniform proof that could shed light on the mysterious relation between the combinatorics of $\mathrm{NC}(W)$ and the invariant theory of $W$. This is the problem which has motivated this work. Roughly speaking, we will bring a complete geometric (and mainly case-free) understanding of certain specifications of Chapoton's formula. For geometric reasons (that will become clear in Sect. 3.2), we consider strict chains in $\mathrm{NC}(W)$ of a given length, rather than multichains. In any bounded posets, their numbers are related to the numbers of multichains by well-known conversion formulas: basically, they are the coefficients of the Zeta polynomial written in the basis of binomial polynomials (see [25, Chap. 3.11]). An alternative way (more adapted in our work) to look at strict chains in $\mathrm{NC}(W)$ is to consider block factorizations of the Coxeter element $c$ :

Definition 1.1 For $c$ a Coxeter element of $W,\left(w_{1}, \ldots, w_{p}\right)$ is called a block factorization of $c$ if:

- $\forall i, w_{i} \in W-\{1\}$

$-w_{1} \ldots w_{p}=c$;

$-\ell\left(w_{1}\right)+\ldots+\ell\left(w_{p}\right)=\ell(c)$.

The reflection length of $c$ equals the rank of $W$, denoted here by $n$. Thus, the maximal number of factors in a block factorization is $n$. Note that block factorizations of $c$ have the same combinatorics of strict chains of $\mathrm{NC}(W)$ : the partial products $w_{1} \cdots w_{i}$ for $i$ from 1 to $p$ form a strict chain by definition. Thus, using simple computations as explained above, we can reformulate Chapoton's formula in terms of these factorizations (an explicit formula is given in Appendix B of [20]). Proving Chapoton's formula amounts to computing the number of block factorizations in $p$ factors for $p$ from 1 to $n$.

We call reduced decompositions of $c$ the factorizations of $c$ in $n$ reflections, i.e., the most refined block factorizations (the set of such factorizations is usually denoted by $\left.\operatorname{Red}_{R}(c)\right)$. The reformulation implies in particular that the number of reduced decompositions (or, equivalently, the number of maximal strict chains in $\mathrm{NC}(W)$ ) is $n$ ! times the leading coefficient of the Zeta polynomial, that is,

$$
\left|\operatorname{Red}_{R}(c)\right|=\frac{n ! h^{n}}{|W|} .
$$

Note that this particular formula was known long before Chapoton's formula (the real case was dealt with by Deligne in [8]; see [4, Proposition 7.5] for the remaining cases). Once again, even for this specific formula, no case-free proof is known.

In [4], Bessis-crediting discussions with Chapoton-interpreted this integer $n ! h^{n} /|W|$ as the degree of a covering (the Lyashko-Looijenga covering LL) constructed from the discriminant of $W$, and he described effectively the relations between the fibers of this covering and the reduced decompositions of $c$. The aim of 
this paper is to explain how, by studying the map LL in more detail, we can obtain new enumerative results, namely formulas for the number of submaximal factorizations of $c$.

Theorem (see Theorem 5.1 and Corollary 5.4) Let $W$ be an irreducible, wellgenerated complex reflection group of rank $n$. Let $c$ be a Coxeter element of $W$, and $\Lambda$ be a conjugacy class of elements of reflection length 2 in $\mathrm{NC}(W)$. Then:

(a) the number of block factorizations of $c$, made up with $n-2$ reflections and one element in the conjugacy class $\Lambda$, is

$$
\left|\operatorname{FACT}_{n-1}^{\Lambda}(c)\right|=\frac{(n-1) ! h^{n-1}}{|W|} \operatorname{deg} D_{\Lambda},
$$

where $D_{\Lambda}$ is an homogeneous polynomial (in the $n-1$ first fundamental invariants) attached to $\Lambda$, determined by the geometry of the discriminant hypersurface of W (see Sect. 5);

(b) the total number of block factorizations of $c$ in $n-1$ factors (or submaximal factorizations) is

$$
\left|\operatorname{FACT}_{n-1}(c)\right|=\frac{(n-1) ! h^{n-1}}{|W|}\left(\frac{(n-1)(n-2)}{2} h+\sum_{i=1}^{n-1} d_{i}\right) .
$$

The first point is new and is a refinement of the second which was already known: like for the number of reduced decompositions, item (b) is a consequence of Chapoton's formula. The main interest of stating (b) is that the proof obtained here is geometric and almost case-free (we still have to rely on some structural properties of LL proved in [4] case-by-case). The structure of the proof is roughly as follows:

1. we use new geometric properties of the morphism LL to prove the formula of point (a) (Sect. 5.1);

2. we find a uniform way to compute $\sum_{\Lambda} \operatorname{deg} D_{\Lambda}$, using an algebraic study of the Jacobian of LL (Sect. 4.2);

3. we deduce the second formula, since $\left|\operatorname{FACT}_{n-1}(c)\right|=\sum_{\Lambda}\left|\operatorname{FACT}_{n-1}^{\Lambda}(c)\right|$ (Sect. 5.2).

Thus, even if the method used here does not seem easily generalizable to factorizations with fewer blocks, it is a new interesting avenue toward a geometric case-free explanation of Chapoton's formulas.

Remark 1.2 During step (2) of the proof, we recover a formula proved (case-by-case) by K. Saito in [22] for real groups and extend it for complex groups. This concerns the bifurcation locus of the discriminant hypersurface of $W$, the factorization of its equation, and the relation with the factorization of the Jacobian of LL (see Sect. 4.3).

Outline In Sect. 2 we give some backgrounds and notations about complex reflection groups, the noncrossing partition lattice, and block factorizations of a Coxeter element. Section 3 is devoted to the construction and properties of the LyashkoLooijenga covering of type $W$, and in particular its relation with factorizations. 
Section 4 is the core of the proof: we study further the algebraic properties of the morphism LL, we show that it gives rise to a "well-ramified" polynomial extension, and we derive factorizations of its Jacobian and its discriminant into irreducibles. We also list the analogies between the properties of LL extensions and those of Galois extensions. In Sect. 5 we use these results to deduce the announced formulas for the number of submaximal factorizations of a Coxeter element. We conclude in the last section by giving a list of numerical data about these factorizations for each irreducible well-generated complex reflection group.

\section{The noncrossing partition lattice of type $W$ and block factorizations of a Coxeter element}

\subsection{Complex reflection groups}

First we recall some notations and definitions about complex reflection groups. For more details, we refer the reader to the books [11] and [14].

For $V$ a finite-dimensional complex vector space, we call a reflection of GL( $V)$ an automorphism $r$ of $V$ of finite order and such that the invariant space $\operatorname{Ker}(r-1)$ is a hyperplane of $V$ (it is called pseudo-reflection by some authors). We call a complex reflection group a finite subgroup of $\mathrm{GL}(V)$ generated by reflections.

A simple way to construct such a group is to take a finite real reflection group (or, equivalently, a finite Coxeter group together with its natural geometric realization) and to complexify it. There are of course many other examples that cannot be seen in a real space. A complete classification of irreducible complex reflection groups was given by Shephard-Todd in [23]: it consists of an infinite series with three parameters and 34 exceptional groups of small ranks.

Throughout this paper we denote by $W$ a subgroup of GL( $V)$ which is a complex reflection group. Note that for real reflection groups, the results presented here are already interesting (and, most of them, new).

We suppose that $W$ is irreducible of rank $n$ (i.e., the linear action on $V$ is irreducible, and the dimension of $V$ is $n$ ). The group $W$ acts naturally on the polynomial algebra $\mathbb{C}[V]=\mathbb{C}\left[v_{1}, \ldots, v_{n}\right]$, where $\left(v_{1}, \ldots, v_{n}\right)$ denotes a basis for $V$. Chevalley-Shephard-Todd's theorem implies that the invariant algebra $\mathbb{C}[V]^{W}$ is again a polynomial algebra, and it can be generated by $n$ algebraically independent homogeneous polynomials $f_{1}, \ldots, f_{n}$ (called the fundamental invariants). The degrees $d_{1}, \ldots, d_{n}$ of these invariants do not depend on the choices for the $f_{i}$ 's (if we require $d_{1} \leq \ldots \leq d_{n}$ ), and they are called the invariant degrees of $W$. Like for finite Coxeter groups, we will denote by $h$ the highest degree $d_{n}$ (called the Coxeter number of $W$ ).

We will also require that $W$ is well-generated, i.e., it can be generated by $n$ reflections (this is always verified in the real case). Then there exist in $W$ so-called Coxeter elements, which generalize the usual notion of a Coxeter element in finite Coxeter groups.

Definition 2.1 A Coxeter element $c$ of $W$ is an $e^{2 i \pi / h}$-regular element (in the sense of Springer's regularity, see [24]), i.e., it is such that there exists a vector $v$ in $V$, outside the reflecting hyperplanes, such that $c(v)=e^{2 i \pi / h} v$. 
As in the real case, Coxeter elements have reflection length $n$ and form a conjugacy class of $W$.

\subsection{The noncrossing partition lattice of type $W$}

Recall that $R$ denotes the set of all reflections of $W$. For $w$ in $W$, the reflection length (or absolute length) of $w$ is:

$$
\ell(w)=\min \left\{p \in \mathbb{N} \mid \exists r_{1}, \ldots, r_{p} \in R, w=r_{1} \ldots r_{p}\right\} .
$$

This length is not to be confused with the usual length in Coxeter groups (called weak length, relative to the generating set of simple reflections) that can be defined only in the real case.

The noncrossing partition lattice is constructed from the absolute order, which is the natural prefix order for the reflection length:

Definition 2.2 We denote by $\preccurlyeq$ the absolute order on $W$, defined by

$$
u \preccurlyeq v \quad \text { if and only if } \ell(u)+\ell\left(u^{-1} v\right)=\ell(v) .
$$

If $c$ is a Coxeter element of $W$, the noncrossing partition lattice of $(W, c)$ is

$$
\mathrm{NC}(W ; c)=\{w \in W \mid w \preccurlyeq c\} .
$$

Since all the Coxeter elements are conjugate, and the reflection length is invariant under conjugation, the structure of $\mathrm{NC}(W ; c)$ does not depend on the choice of the Coxeter element $c$. Thus we will just write $\mathrm{NC}(W)$ for short, considering $c$ fixed for the rest of the paper. In the prototypal case of type $A$, where $W$ is the symmetric group $\mathfrak{S}_{n+1}, R$ is the set of all transpositions, and $c$ is an $(n+1)$-cycle; then $\mathrm{NC}(W)$ is isomorphic to the set of noncrossing partitions of an $(n+1)$-gon, as introduced by Kreweras [13]. In general, the noncrossing partition lattice of type $W$ has a very rich combinatorial structure; we refer to Chap. 1 of [1] or the introduction of [20].

\subsection{Multichains in $\mathrm{NC}(W)$ and block factorizations of a Coxeter element}

Recall from Definition 1.1 that a block factorization of $c$ is a factorization in nontrivial factors, such that the lengths of the factors add up to the length of $c$ (i.e., there exist reduced decompositions of $c$ obtained from concatenation of reduced decompositions of the blocks).

We denote by $\operatorname{FACT}(c)$ (resp. $\operatorname{FACT}_{p}(c)$ ) the set of block factorizations of $c$ (resp. factorizations in $p$ factors). Note that the length of $c$ is equal to the rank $n$ of $W$, so any block factorization of $c$ determines a composition (ordered partition) of the integer $n$. The set $\mathrm{FACT}_{n}(c)$ corresponds to the set of reduced decompositions of $c$ into reflections, usually denoted by $\operatorname{Red}_{R}(c)$ (composition $(1,1, \ldots, 1)$ ).

To simplify we will write, from now on, factorization for block factorization.

If $\left(w_{1}, \ldots, w_{p}\right)$ is a factorization of $c$, then we canonically get a (strict) chain in $\mathrm{NC}(W)$ :

$$
w_{1} \prec w_{1} w_{2} \prec \ldots \prec w_{1} \ldots w_{p}=c .
$$


Strict chains are related to multichains by known formulas, so that we can pass from enumeration of multichains in $\mathrm{NC}(W)$ to enumeration of factorizations of $c$, and vice versa (see for example [20, Appendix B] or [25, Chap. 3.11]).

In the following section, we describe a geometric construction of these factorizations, and how they are related to the fibers of a topological covering.

\section{Lyashko-Looijenga covering and factorizations of a Coxeter element}

\subsection{Discriminant of a well-generated reflection group and Lyashko-Looijenga covering}

Let $W$ be a well-generated, irreducible complex reflection group with invariant polynomials $f_{1}, \ldots, f_{n}$, homogeneous of degrees $d_{1} \leq \ldots \leq d_{n}=h$. Note that the quotient-space ${ }^{1} W \backslash V$ is then isomorphic to $\mathbb{C}^{n}$ :

$$
\begin{aligned}
W \backslash V & \stackrel{\sim}{\rightarrow} \mathbb{C}^{n} \\
\bar{v} & \mapsto\left(f_{1}(v), \ldots, f_{n}(v)\right) .
\end{aligned}
$$

We recall here the construction of the Lyashko-Looijenga map of type $W$ (for more details, see [4, Sect. 5] or [21, Sect. 3]).

Let us denote by $\mathscr{A}$ the set of all reflecting hyperplanes of $W$ and consider the discriminant of $W$ defined by

$$
\Delta_{W}:=\prod_{H \in \mathscr{A}} \alpha_{H}^{e_{H}},
$$

where $\alpha_{H}$ is an equation of $H$, and $e_{H}$ is the order of the parabolic subgroup $W_{H}=\operatorname{Fix}(H)$. The discriminant lies in $\mathbb{C}[V]^{W}=\mathbb{C}\left[f_{1}, \ldots, f_{n}\right]$, and it is an equation for the discriminant hypersurface

$$
\mathscr{H}:=W \backslash \bigcup_{H \in \mathscr{A}} H \subseteq W \backslash V \simeq \mathbb{C}^{n} .
$$

It is known (see [4, Theorem 2.4]) that when $W$ is well-generated, the fundamental invariants $f_{1}, \ldots, f_{n}$ can be chosen such that the discriminant of $W$ is a monic polynomial of degree $n$ in $f_{n}$ of the form

$$
\Delta_{W}=f_{n}^{n}+a_{2} f_{n}^{n-2}+\ldots+a_{n},
$$

where $a_{i} \in \mathbb{C}\left[f_{1}, \ldots, f_{n-1}\right]$. This property implies that if we fix $f_{1}, \ldots, f_{n-1}$, then $\Delta_{W}$ always has $n$ roots (counting multiplicities) as a polynomial in $f_{n}$.

Let us define $Y:=\operatorname{Spec} \mathbb{C}\left[f_{1}, \ldots, f_{n-1}\right] \simeq \mathbb{C}^{n-1}$, so that $W \backslash V \simeq Y \times \mathbb{C}$. Then the geometric version of the property given above is that the intersection of the hypersurface $\mathscr{H}$ with the complex line $\left\{\left(y, f_{n}\right) \mid f_{n} \in \mathbb{C}\right\}$ (for a fixed $y \in Y$ ) generically has cardinality $n$. The definition of the Lyashko-Looijenga map comes from these observations.

\footnotetext{
${ }^{1}$ The action of $W$ on $V$ is conventionally on the left side, so we prefer to write the quotient-space $W \backslash V$.
} 
Definition 3.1 We denote by $E_{n}$ the set of centered configurations of $n$ points in $\mathbb{C}$, i.e.,

$$
E_{n}:=H_{0} / \mathfrak{S}_{n}, \quad \text { where } H_{0}=\left\{\left(x_{1}, \ldots, x_{n}\right) \in \mathbb{C}^{n} \mid \sum_{i=1}^{n} x_{i}=0\right\} .
$$

The Lyashko-Looijenga map of type $W$ is defined by

$$
\begin{aligned}
Y & \stackrel{\mathrm{LL}}{\longrightarrow} E_{n} \\
y=\left(f_{1}, \ldots, f_{n-1}\right) & \mapsto \text { multiset of roots of } \Delta_{W}\left(f_{1}, \ldots, f_{n}\right) \text { in the variable } f_{n} .
\end{aligned}
$$

Remark 3.2 We can also regard LL as an algebraic morphism. Indeed, the natural coordinates for $E_{n}$ as an algebraic variety are the $n-1$ elementary symmetric polynomials $e_{2}\left(x_{1}, \ldots, x_{n}\right), \ldots, e_{n}\left(x_{1}, \ldots, x_{n}\right)$. Thus, the algebraic version of the map LL is (up to some unimportant signs) simply the morphism

$$
\begin{array}{cl}
\mathbb{C}^{n-1} & \rightarrow \\
\left(f_{1}, \ldots, f_{n-1}\right) & \mapsto\left(a_{2}\left(f_{1}, \ldots, f_{n-1}\right), \ldots, a_{n}\left(f_{1}, \ldots, f_{n-1}\right)\right) .
\end{array}
$$

To shorten the notations, we will also denote this morphism by LL, whenever in an algebraic context (mainly in Sect. 4).

We denote by $E_{n}^{\text {reg }}$ the set of configurations in $E_{n}$ with $n$ distinct points, and we define the bifurcation locus of LL, namely $\mathscr{K}:=\mathrm{LL}^{-1}\left(E_{n}-E_{n}^{\mathrm{reg}}\right)$. Equivalently, we have

$$
\mathscr{K}:=\left\{y \in Y \mid D_{\mathrm{LL}}(y)=0\right\},
$$

where $D_{\mathrm{LL}}$ is called the LL-discriminant and is defined by

$$
D_{\mathrm{LL}}:=\operatorname{Disc}\left(\Delta_{W}\left(y, f_{n}\right) ; f_{n}\right) \in \mathbb{C}\left[f_{1}, \ldots, f_{n-1}\right] .
$$

Example 3.3 The picture of Fig. 1 gives a simplified geometric view of what happens for the group $W\left(A_{3}\right)$. The discriminant hypersurface $\mathscr{H}$ and the bifurcation locus $\mathscr{K}$ are described. The map LL associates to any point in $Y$ the multiset of intersection points of the line $\{(y, x) \mid x \in \mathbb{C}\}$ (vertical green lines ) with $\mathscr{H}$ (yellow points).

The first important property is the following (from [4, Theorem 5.3]):

Property (P0) The restriction of LL : Y $-\mathscr{K} \rightarrow E_{n}^{\mathrm{reg}}$ is a topological covering of degree $\frac{n ! h^{n}}{|W|}$.

We call this integer the Lyashko-Looijenga number of type W.

\subsection{Geometric construction of factorizations}

Before explaining the construction of factorizations from the discriminant hypersurface, we recall some useful properties of the geometric stratification associated to the parabolic subgroups of $W$. 
Fig. 1 Example of $W\left(A_{3}\right)$. The picture represents a fragment of the real part of the discriminant hypersurface $\mathscr{H}$ (equation $\operatorname{Disc}\left(T^{4}+f_{1} T^{2}-f_{2} T+f_{3} ; T\right)=0$, called the swallowtail hypersurface), as well as its bifurcation locus $\mathscr{K}$. The vertical is chosen to be the direction of $f_{n}$. The other information is described gradually in Examples 3.3, 3.5, and 4.4

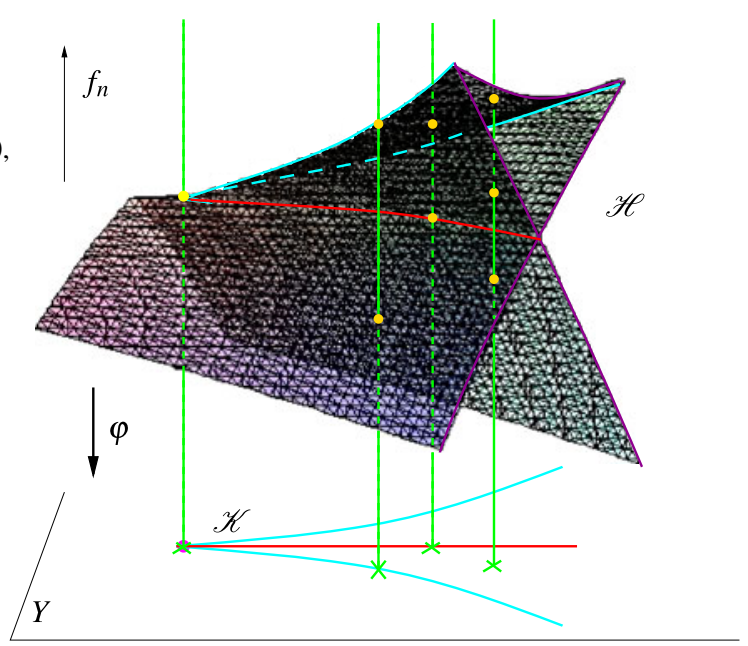

Discriminant stratification The space $V$, together with the hyperplane arrangement $\mathscr{A}$, admits a natural stratification by the flats, namely, the elements of the intersection lattice $\mathscr{L}:=\left\{\bigcap_{H \in \mathscr{B}} H \mid \mathscr{B} \subseteq \mathscr{A}\right\}$.

As the $W$-action on $V$ maps a flat to a flat, this stratification gives rise to a quotient stratification $\overline{\mathscr{L}}$ of $W \backslash V$ :

$$
\overline{\mathscr{L}}=W \backslash \mathscr{L}=(p(L))_{L \in \mathscr{L}}=(W \cdot L)_{L \in \mathscr{L}},
$$

where $p$ is the projection $V \rightarrow W \backslash V$. For each stratum $\Lambda$ in $\overline{\mathscr{L}}$, we denote by $\Lambda^{0}$ the complement in $\Lambda$ of the union of the strata strictly included in $\Lambda$. The family $\left(\Lambda^{0}\right)_{\Lambda \in \overline{\mathscr{L}}}$ forms an open stratification of $W \backslash V$, called the discriminant stratification.

There is a natural bijection between the set of flats in $V$ and the set of parabolic subgroups of $W$ (Steinberg's theorem). By quotienting by the action of $W$, this leads to other descriptions of the stratification $\overline{\mathscr{L}}$ :

Proposition 3.4 The set $\overline{\mathscr{L}}$ is in canonical bijection with:

- the set of conjugacy classes of parabolic subgroups of $W$;

- the set of conjugacy classes of parabolic Coxeter elements (i.e., Coxeter elements of parabolic subgroups);

- the set of conjugacy classes of elements of $\mathrm{NC}(W)$.

Through these bijections, the codimension of a stratum $\Lambda$ corresponds to the rank of the associated parabolic subgroup and to the reflection length of the parabolic Coxeter element.

We refer to [21, Sect. 6] for details and proofs.

Example 3.5 In the picture of Fig. 1, the two strata of $\overline{\mathscr{L}}$ of codimension 2 are drawn in red and blue (the blue one is the one forming a cusp). Through the bijection of 
Proposition 3.4, the blue one corresponds to the conjugacy class of a parabolic Coxeter element of type $A_{2}$ (viewed in $\mathfrak{S}_{4}$, this is a 3-cycle), and the red one corresponds to the conjugacy class of a parabolic Coxeter element of type $A_{1} \times A_{1}$ (i.e., a product of two commuting transpositions in $\mathfrak{S}_{4}$ ).

Geometric factorizations and compatibilities In [21] we established a way to construct factorizations geometrically from the discriminant hypersurface $\mathscr{H}$. We describe below the idea of the construction and some of its properties; for details and proofs, see [21, Sect. 4] and [4, Sect. 6].

The starting point is the construction of a map

$$
\begin{aligned}
\rho: \mathscr{H} & \rightarrow W \\
(y, x) & \mapsto c_{y, x},
\end{aligned}
$$

by the following steps (note that $(y, x)$ lies in $\mathscr{H}$ if and only if the multiset LL( $y$ ) contains $x$ ).

1. Consider a small loop in $\mathbb{C}^{n}-\mathscr{H}$, which always stays in the fiber $\{(y, t), t \in \mathbb{C}\}$, and which turns once around $x$ (but not around any other $x^{\prime}$ in $\operatorname{LL}(y)$ ).

2. This loop determines an element $b_{y, x}$ of $\pi_{1}\left(\mathbb{C}^{n}-\mathscr{H}\right)=\pi_{1}\left(V^{\text {reg }} / W\right)$, which is the braid group $B(W)$ of $W$.

3. Send $b_{y, x}$ to $c_{y, x}$ via a fixed surjection $B(W) \rightarrow W$.

The map $\rho$ has the following fundamental properties.

Property (P1) If $\left(x_{1}, \ldots, x_{p}\right)$ is the ordered support of $\mathrm{LL}(y)$ (for the lexicographical order on $\left.\mathbb{C} \simeq \mathbb{R}^{2}\right)$, then the $p$-tuple $\left(c_{y, x_{1}}, \ldots, c_{y, x_{p}}\right)$ lies in $\operatorname{FACT}_{p}(c)$.

Property (P2) For all $x \in \mathrm{LL}(y), c_{y, x}$ is a parabolic Coxeter element; its length is equal to the multiplicity of $x$ in $\operatorname{LL}(y)$, and its conjugacy class corresponds (via the bijection of Proposition 3.4) to the unique stratum $\Lambda$ in $\overline{\mathscr{L}}$ such that $(y, x) \in \Lambda^{0}$.

According to Property (P1), we call the tuple $\left(c_{y, x_{1}}, \ldots, c_{y, x_{p}}\right)$ (where $\left(x_{1}, \ldots, x_{p}\right)$ is the ordered support of $\operatorname{LL}(y))$ the factorization of $c$ associated to $y$, and we denote it by facto $(y)$.

Any block factorization determines a composition of $n$. To any configuration of $E_{n}$ we can also associate a composition of $n$, formed by the multiplicities of its elements in the lexicographical order. Then Property (P2) implies that for any $y$ in $Y$, the compositions associated to $\operatorname{LL}(y)$ and facto $(y)$ are the same. The third fundamental property (see [21, Theorem 5.1] or [4, Theorem 7.9]) is the following.

Property (P3) The map $\mathrm{LL} \times$ facto $: Y \rightarrow E_{n} \times \operatorname{FACT}(c)$ is injective, and its image is the entire set of compatible pairs (i.e., pairs with same associated composition).

In other words, for each $y \in Y$, the fiber $\operatorname{LL}^{-1}(\operatorname{LL}(y))$ is in bijection (via facto) with the set of factorizations whose associated composition of $n$ is the same as that associated to facto $(y)$. This fundamental property is a reformulation of a theorem by Bessis; the proof still relies on some case-by-case analysis. 


\section{Lyashko-Looijenga extensions}

Property (P3) is particularly helpful to compute algebraically certain classes of factorizations. For example, if $y$ lies in $Y-\mathscr{K}$, then facto $(y)$ is in $\operatorname{FACT}_{n}(c)$ (in other words, it is a reduced decomposition of $c$ ), i.e., the associated composition is $(1,1, \ldots, 1)$. Thus, from $(\mathrm{P} 3)$, the set $\operatorname{Red}_{R}(c)$ is in bijection with any generic fiber of LL (the fiber of any point in $E_{n}^{\text {reg }}$ ), so it has cardinality $n ! h^{n} /|W|$, because of Property (P0). Note that this number has been computed algebraically, using the fact that the algebraic morphism LL is "weighted-homogeneous."

In order to go further and count more complicated factorizations of $c$, we need a more precise algebraic study of the morphism LL, in particular its restriction to the bifurcation locus $\mathscr{K}$.

\subsection{Ramification locus for LL}

Let us first explain the reason why LL is étale on $Y-\mathscr{K}$ (as stated in Property (P0)), where we recall:

$$
\mathscr{K}=\{y \in Y \mid \text { the multiset } \operatorname{LL}(y) \text { has multiple points }\} .
$$

The argument goes back to Looijenga [15] and is used without details in the proof of Lemma 5.6 of [4].

We begin with a more general setting. Let $n \geq 1$, and $P \in \mathbb{C}\left[T_{1}, \ldots, T_{n}\right]$ of the form

$$
P=T_{n}^{n}+a_{2}\left(T_{1}, \ldots, T_{n-1}\right) T_{n}^{n-2}+\ldots+a_{n}\left(T_{1}, \ldots T_{n-1}\right)
$$

(here the polynomials $a_{i}$ do not need to be quasi-homogeneous). As in the case of LL, we define the hypersurface $\mathscr{H}:=\{P=0\} \subseteq \mathbb{C}^{n}$ and a map $\psi: \mathbb{C}^{n-1} \rightarrow E_{n}$, sending $y=\left(T_{1}, \ldots, T_{n-1}\right) \in \mathbb{C}^{n-1}$ to the multiset of roots of $P\left(y, T_{n}\right)$ (as a polynomial in $\left.T_{n}\right)$. This map can also be considered as the morphism $y \mapsto\left(a_{2}(y), \ldots, a_{n}(y)\right)$.

We set

$$
J_{\psi}(y)=\operatorname{Jac}\left(\left(a_{2}, \ldots, a_{n}\right) / y\right)=\operatorname{det}\left(\frac{\partial a_{i}}{\partial T_{j}}\right)_{\substack{1 \leq i \leq n \\ 1 \leq j \leq n-1}} .
$$

Proposition 4.1 (after Looijenga) With the notations above, let y be a point in $\mathbb{C}^{n-1}$, with $\psi(y)$ being the multiset $\left\{x_{1}, \ldots, x_{n}\right\}$. Suppose that the $x_{i}$ 's are pairwise distinct.

Then the points $\left(y, x_{i}\right)$ are regular on $\mathscr{H}$. Moreover, the $n$ hyperplanes tangent to $\mathscr{H}$ at $\left(y, x_{1}\right), \ldots,\left(y, x_{n}\right)$ are in general position if and only if $J_{\psi}(y) \neq 0$ (i.e., $\psi$ is étale at $y)$.

Proof Let $\alpha$ be a point in $\mathscr{H}$. If it exists, the hyperplane tangent to $\mathscr{H}$ at $\alpha$ is directed by its normal vector $\operatorname{grad}_{\alpha} P=\left(\frac{\partial P}{\partial T_{1}}(\alpha), \ldots, \frac{\partial P}{\partial T_{n}}(\alpha)\right)$.

Let $y$ be a point in $\mathbb{C}^{n-1}$ such that the associated $x_{i}$ 's are pairwise distinct. Then the polynomial in $T_{n}, P\left(y, T_{n}\right)$, has the $x_{i}$ 's as simple roots, so for each $i$, $\frac{\partial P}{\partial T_{n}}\left(y, x_{i}\right) \neq 0$, and the point $\left(y, x_{i}\right)$ is regular on $\mathscr{H}$. 
The tangent hyperplanes associated to $y$ are in general position if and only if $\operatorname{det} M_{y} \neq 0$, where $M_{y}$ is the matrix with columns

$$
\left(\operatorname{grad}_{\left(y, x_{1}\right)} P ; \ldots ; \operatorname{grad}_{\left(y, x_{n}\right)} P\right) .
$$

After computation, we get $M_{y}=A_{y} V_{y}$, where

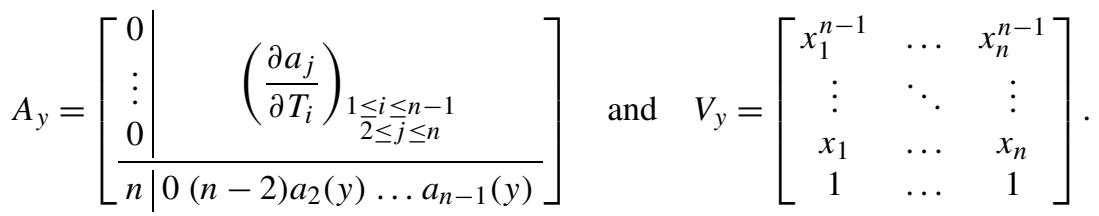

As the $x_{i}$ 's are distinct, the Vandermonde matrix $V_{y}$ is invertible. As $\operatorname{det} A_{y}=$ $n J_{\psi}(y)$, we can conclude that $\operatorname{det} M_{y} \neq 0$ if and only if $J_{\psi}(y) \neq 0$.

If the $x_{i}$ 's are not distinct, nothing can be said in general. But if $\psi$ is a LyashkoLooijenga morphism LL, then we can deduce the following property.

Corollary 4.2 Let $y$ be a point in $\mathbb{C}^{n-1}$, and suppose that $\operatorname{LL}(y)$ contains $n$ distinct points. Then $J_{\mathrm{LL}}(y) \neq 0$.

In other words, LL is étale on (at least) $Y-\mathscr{K}$.

Proof Set $\operatorname{LL}(y)=\left\{x_{1}, \ldots, x_{n}\right\}$. As the $x_{i}$ 's are distinct, from Lemma 4.1 one has to study the hyperplanes tangent to $\mathscr{H}$ at $\left(y, x_{1}\right), \ldots,\left(y, x_{n}\right)$. By using their characterization in terms of basic derivations of $W$, it is straightforward to show that the $n$ hyperplanes are always in general position: we refer to the proof of [4, Lemma 5.6].

In the following we will prove the equality $Z\left(J_{\mathrm{LL}}\right)=\mathscr{K}$, i.e., that $\mathrm{LL}$ is étale exactly on $Y-\mathscr{K}$.

4.2 The well-ramified property for LL

Following Remark 3.2, consider LL as the algebraic morphism

$$
\begin{aligned}
\mathbb{C}^{n-1} & \rightarrow \\
\left(f_{1}, \ldots, f_{n-1}\right) & \mapsto\left(a_{2}\left(f_{1}, \ldots, f_{n-1}\right), \ldots, a_{n}\left(f_{1}, \ldots, f_{n-1}\right)\right) .
\end{aligned}
$$

According to [4, Theorem 5.3], this is a finite quasi-homogeneous map (for the weights $\left.\operatorname{deg}\left(f_{i}\right)=d_{i}, \operatorname{deg} a_{j}=j h\right)$. So we get a graded finite polynomial extension

$$
A=\mathbb{C}\left[a_{2}, \ldots, a_{n}\right] \subseteq \mathbb{C}\left[f_{1}, \ldots, f_{n-1}\right]=B .
$$

Such extensions are studied in [19]. Let us recall the properties and definitions that we need. For such an extension $A \subseteq B$, we denote by $\operatorname{Spec}_{1}(B)$ the set of ideals of $B$ of height one, and $\operatorname{Spec}_{1}^{\mathrm{ram}}(B)$ its subset consisting of ideals which are ramified 
over $A$. These ideals are principal, and we will talk about "the set of ramified polynomials of the extension" for a set of representatives of generators of these ramified ideals.

In [19, Theorem 1.8] we described the factorization of the Jacobian polynomial of the extension $J_{B / A}$. We can apply it here and obtain:

$$
J_{\mathrm{LL}}=\operatorname{det}\left(\frac{\partial a_{i}}{\partial f_{j}}\right)_{\substack{2 \leq i \leq n \\ 1 \leq j \leq n-1}} \doteq \prod_{Q \in \operatorname{Spec}_{1}^{\mathrm{ram}}(B)} Q^{e-1},
$$

where $e_{Q}$ is the ramification index of $Q$ (and $\doteq$ designates equality up to a scalar).

We also introduced in [19] the notion af a well-ramified extension:

Definition 4.3 A finite graded polynomial extension $A \subseteq B$ is well-ramified if

$$
\left(J_{B / A}\right) \cap A=\left(\prod_{Q \in \operatorname{Spec}_{1}^{\text {ram }}(B)} Q^{e}\right) \text { as an ideal of } A .
$$

Well-ramified extensions are generalizations of Galois extensions (where $A$ is the algebra of invariants of $B$ under the action of a reflection group) but keep some of their characteristics. We refer to [19, Sect. 3.2] for details and other characterizations of this property. The name "well-ramified" is chosen accordingly to one of these characterizations, namely:

"For any $\mathfrak{p} \in \operatorname{Spec}_{1}(A)$, if there exists $\mathfrak{q}_{0} \in \operatorname{Spec}_{1}(B)$ over $\mathfrak{p}$ which is ramified, then any other $\mathfrak{q}$ in $\operatorname{Spec}_{1}(B)$ over $\mathfrak{p}$ is also ramified." ([19, Proposition 3.2 (iv)])

In the following of this subsection we prove that the extension defined by LL is well-ramified. In Sect. 4.4 we will compare the setting of Lyashko-Looijenga extensions to that of Galois extensions.

We recall from Sect. 3.1 the definition of $D_{\mathrm{LL}}$ :

$$
D_{\mathrm{LL}}:=\operatorname{Disc}\left(f_{n}^{n}+a_{2} f_{n}^{n-2}+\ldots+a_{n} ; f_{n}\right),
$$

so that $\mathscr{K}=\mathrm{LL}^{-1}\left(E_{n}-E_{n}^{\mathrm{reg}}\right)$ is the zero locus of $D_{\mathrm{LL}}$ in $Y$. We denote by $\overline{\mathscr{L}_{2}}$ the set of all closed strata in $\overline{\mathscr{L}}$ of codimension 2 . Note that $\overline{\mathscr{L}_{2}}$ is also the set of conjugacy classes of elements of $\mathrm{NC}(W)$ of length 2 (cf. Proposition 3.4).

We define the following map:

$$
\begin{aligned}
& \varphi: W \backslash V \simeq Y \times \mathbb{C} \rightarrow Y \\
& \bar{v}=(y, x) \quad \mapsto y .
\end{aligned}
$$


Then, using the notations and properties of Sect. 3.2, we have:

$$
\begin{aligned}
y \in \mathscr{K} & \Leftrightarrow \exists x \in \operatorname{LL}(y) \text { with multiplicity } \geq 2 \\
& \Leftrightarrow \exists x \in \operatorname{LL}(y) \text { such that } \ell\left(c_{y, x}\right) \geq 2 \\
& \Leftrightarrow \quad \exists x \in \operatorname{LL}(y) \text { such that }(y, x) \in \Gamma^{0} \text { for some stratum } \Gamma \in \overline{\mathscr{L}} \\
& \Leftrightarrow \quad \text { of } \operatorname{codim} . \geq 2 \\
& \Leftrightarrow \quad \exists \Lambda \in \operatorname{LL}(y), \exists \Lambda \in \overline{\mathscr{L}_{2}} \text { such that }(y, x) \in \Lambda
\end{aligned}
$$

So the hypersurface $\mathscr{K}$ is the union of the $\varphi(\Lambda)$ for $\Lambda \in \overline{\mathscr{L}_{2}}$. It can be shown that they are in fact its irreducible components (cf. [21, Proposition 7.4]). Thus we can write

$$
D_{\mathrm{LL}}=\prod_{\Lambda \in \overline{\mathscr{L}_{2}}} D_{\Lambda}^{r_{\Lambda}}
$$

for some $r_{\Lambda} \geq 1$, where the $D_{\Lambda}$ are irreducible (homogeneous) polynomials in $B=\mathbb{C}\left[f_{1}, \ldots, f_{n-1}\right]$ such that $\varphi(\Lambda)=\left\{D_{\Lambda}=0\right\}$.

Example 4.4 In the example of $A_{3}$ in Fig. 1, the two strata of $\overline{\mathscr{L}_{2}}$ described in Example 3.5 - let us call them $\Lambda_{\text {red }}$ and $\Lambda_{\text {blue }}$-project (by $\varphi$ ) onto the two irreducible components of $\mathscr{K}$. The explicit computation gives that the power $r_{\Lambda_{\text {red }}}$ of $D_{\Lambda_{\text {red }}}$ (resp. for blue) in $D_{\mathrm{LL}}$ equals 2 (resp. 3), which is also the common order of parabolic Coxeter elements in the conjugacy class corresponding to the strata (indeed, those are products of two commuting transpositions, resp. 3-cycles). This turns out to be a general phenomenon, as described in the following theorem.

Now we give an important interpretation of the integers $r_{\Lambda}$ and deduce that LL is a well-ramified extension.

Theorem 4.5 Let LL be the Lyashko-Looijenga extension associated to a wellgenerated, irreducible complex reflection group, together with the above notations. For any $\Lambda$ in $\overline{\mathscr{L}_{2}}$, let $w$ be a (length 2) parabolic Coxeter element of $W$ in the conjugacy class corresponding to $\Lambda$. Recall that $r_{\Lambda}$ denotes the power of $D_{\Lambda}$ in $D_{\mathrm{LL}}$. Then:

(a) The integer $r_{\Lambda}$ is the number of reduced decompositions of $w$ into two reflections. When $W$ is a 2-reflection group, ${ }^{2}$ it is simply the order of $w$.

(b) The set of ramified polynomials of the extension $A \subseteq B$ is the family $\left\{D_{\Lambda} \mid \Lambda \in\right.$ $\left.\overline{\mathscr{L}_{2}}\right\}$, and the ramification index of $D_{\Lambda}$ is $r_{\Lambda}$.

(c) The LL-Jacobian satisfies: $J_{\mathrm{LL}} \doteq \prod_{\Lambda \in \overline{\mathscr{L}}_{2}} D_{\Lambda}^{r_{\Lambda}-1}$.

(d) The "LL-discriminant" $D_{\mathrm{LL}}=\prod_{\Lambda \in \overline{\mathscr{L}}_{2}} D_{\Lambda}^{r_{\Lambda}}$ is a generator for the ideal $\left(J_{\mathrm{LL}}\right) \cap A$.

(e) The polynomial extension associated to LL is well-ramified.

\footnotetext{
${ }^{2}$ A 2-reflection group is a complex reflection group generated by reflections of order 2; see [4, Theorem 2.2] for an interesting property of these groups.
} 
Proof Let us prove first that all the ramified polynomials in $B$ are included in $\left\{D_{\Lambda} \mid \Lambda \in \overline{\mathscr{L}}_{2}\right\}$. The polynomial $D_{\mathrm{LL}}$ is irreducible in $A$ since, as a polynomial in $a_{2}, \ldots, a_{n}$, it is the discriminant of a reflection group of type $A_{n-1}$. Therefore, for all $\Lambda$ in $\overline{\mathscr{L}_{2}}$, the inclusion

$$
\left(D_{\Lambda}\right) \cap A \supseteq(D)
$$

is an inclusion between prime ideals of height one in $A$. So we have $\left(D_{\Lambda}\right) \cap A=(D)$, and the ramification index $e_{D_{\Lambda}}$ is equal to $v_{D_{\Lambda}}(D)=r_{\Lambda}$. According to Corollary 4.2, if $J_{\mathrm{LL}}(y)=0$, then $\operatorname{LL}(y) \notin E_{n}^{\mathrm{reg}}$. So the variety of zeros of $J_{\mathrm{LL}}$ (defined by the ramified polynomials in $B$ ) is included in the preimage

$$
\mathrm{LL}^{-1}(Z(D))=\bigcup_{\Lambda \in \overline{\mathscr{L}}_{2}} Z\left(D_{\Lambda}\right) .
$$

Thus, any ramified polynomial of the extension is necessarily one of the $D_{\Lambda}$ 's.

Let us prove the point $(a)$. Let $\Lambda$ be a stratum in $\overline{\mathscr{L}_{2}}$, and $\mu$ be the composition $(2,1, \ldots, 1)$ of $n$. Choose $\xi=\left(w, s_{3}, \ldots, s_{n}\right)$ in $\operatorname{FACT}_{\mu}(c)$ such that the conjugacy class of $w$ (the only element of length 2 in $\xi$ ) corresponds to $\Lambda$. Fix $e \in E_{n}$ with composition type $\mu$ and such that the real parts of its support are distinct. There exists a unique $y_{0}$ in $Y$ such that $\operatorname{LL}\left(y_{0}\right)=e$ and facto $\left(y_{0}\right)=\xi$ (by Property (P3) in Sect. 3.2). Moreover $y_{0}$ lies in $\varphi(\Lambda)$ (Property (P2)). Using the precise definition of the map facto [21, Definition 4.2], and the "Hurwitz rule" [21, Lemma 4.5], we deduce that for a sufficiently small connected neighborhood $\Omega_{0}$ of $y_{0}$, if $y$ is in $\Omega_{0} \cap$ $(Y-\mathscr{K})$, then facto $(y)$ is in

$$
F_{w}:=\left\{\left(s_{1}^{\prime}, s_{2}^{\prime}, \ldots, s_{n}^{\prime}\right) \in \operatorname{Red}_{R}(c) \mid s_{1}^{\prime} s_{2}^{\prime}=w \text { and } s_{i}^{\prime}=s_{i} \forall i \geq 3\right\} .
$$

Let us fix $y$ in $\Omega_{0} \cap(Y-\mathscr{K})$. Then, because of Property (P3), we get an injection

$$
\text { facto }: \mathrm{LL}^{-1}(\mathrm{LL}(y)) \cap \Omega_{0} \hookrightarrow F_{w} \text {. }
$$

But this map is also surjective, thanks to the covering properties of LL and the transitivity of the Hurwitz action on $w$. Indeed, we can "braid" $s_{1}^{\prime}$ and $s_{2}^{\prime}$ (by cyclically intertwining the two corresponding points of $\operatorname{LL}(y)$, while staying in the neighborhood) so as to obtain any factorization of $w$. Thus,

$$
\left|\operatorname{LL}^{-1}(\operatorname{LL}(y)) \cap \Omega_{0}\right|=\left|F_{w}\right| .
$$

Using the classical characterization of the ramification index (see, e.g., [19, Proposition 2.4]), we infer that $\left|F_{w}\right|$ is equal to the ramification index $e_{D_{\Lambda}}$, so $r_{\Lambda}=\left|F_{w}\right|$. This is also the number of reduced decompositions of $w$, i.e., the Lyashko-Looijenga number for the parabolic subgroups in the conjugacy class $\Lambda$.

For any rank 2 parabolic subgroup with degrees $d_{1}^{\prime}, h^{\prime}$, the LL-number is $2 h^{\prime} / d_{1}^{\prime}$. In the particular case where $W$ is a 2-reflection group, such a subgroup is a dihedral group, hence $d_{1}^{\prime}$ equals 2 , and $r_{\Lambda}$ is the order $h^{\prime}$ of the associated parabolic Coxeter element $w$. 
Consequently, for all $\Lambda \in \overline{\mathscr{L}_{2}}, e_{D_{\Lambda}}=r_{\Lambda}$ is strictly greater than 1 , so $D_{\Lambda}$ is ramified, and statement $(b)$ is proven. Using formula $(*)$ above, this also directly implies $(c)$.

Moreover, we obtain:

$$
\prod_{Q \in \operatorname{Spec}_{1}^{\mathrm{ram}}(B)} Q^{e_{Q}}=\prod_{\Lambda \in \mathscr{L}_{2}} D_{\Lambda}^{e_{D_{\Lambda}}}=D_{\mathrm{LL}}
$$

so this polynomial lies in $A$. We recognize one of the characterizations of a wellramified extension (namely [19, Proposition 3.2(iii)]), from which we deduce $(d)$ and $(e)$.

\subsection{A more intrinsic definition of the Lyashko-Looijenga Jacobian}

In this subsection we give an alternate definition for the Jacobian $J_{\mathrm{LL}}$, which is more intrinsic, and which allows us to recover a formula observed by K. Saito.

We will use the following elementary property. Suppose that $P \in$ $\mathbb{C}\left[T_{1}, \ldots, T_{n-1}, X\right]$ has the form

$$
P=X^{n}+b_{1} X^{n-1}+\ldots+b_{n}
$$

with $b_{1}, \ldots, b_{n} \in \mathbb{C}\left[T_{1}, \ldots, T_{n-1}\right]$. Note that we do not require $b_{1}$ to be zero. Let us denote by $J(P)$ the polynomial

$$
J(P):=\operatorname{Jac}\left(\left(P, \frac{\partial P}{\partial X}, \ldots, \frac{\partial^{n-1} P}{\partial X^{n-1}}\right) /\left(T_{1}, \ldots, T_{n-1}, X\right)\right) .
$$

Lemma 4.6 Let $P$ be as above. We set $Y=X+\frac{b_{1}}{n}$ and denote by $Q$ the polynomial in $\mathbb{C}\left[T_{1}, \ldots, T_{n-1}, Y\right]$ such that $Q\left(T_{1}, \ldots, T_{n-1}, Y\right)=P\left(T_{1}, \ldots, T_{n-1}, X\right)$. We consider the polynomials $a_{2}, \ldots, a_{n}$ in $\mathbb{C}\left[T_{1}, \ldots, T_{n-1}\right]$ such that $Q=Y^{n}+a_{2} Y^{n-2}+$ $\ldots+a_{n}$.

We define $J(P)$ as above and $J(Q)$ similarly $(Y$ replacing $X)$. Then:

(i) $J(P)=J(Q)$;

(ii) $J(P)$ does not depend on $X$, and $J(P) \doteq \mathrm{Jac}\left(\left(a_{2}, \ldots, a_{n}\right) /\left(T_{1}, \ldots, T_{n-1}\right)\right)$.

The proof is elementary and can be found in [20, Lemma 3.4]. Consequently, we have an intrinsic definition for the Lyashko-Looijenga Jacobian:

$$
J_{\mathrm{LL}} \doteq J\left(\Delta_{W}\right)=\operatorname{Jac}\left(\left(\Delta_{W}, \frac{\partial \Delta_{W}}{\partial f_{n}}, \ldots, \frac{\partial^{n-1} \Delta_{W}}{\partial f_{n}^{n-1}}\right) /\left(f_{1}, \ldots, f_{n}\right)\right),
$$

where $f_{1}, \ldots, f_{n}$ do not need to be chosen such that the coefficient of $f_{n}^{n-1}$ in $\Delta_{W}$ is zero. Note that for the computation of $D_{\mathrm{LL}}$ as well, the fact that the coefficient $a_{1}$ is zero in $\Delta_{W}$ is not important, because of invariance by translation.

With these alternative definitions, the factorization of the Jacobian given by Theorem 4.5 has already been observed (for real groups) by Kyoji Saito: it is formula 2.2.3 in [22]. He uses this formula in his study of the semi-algebraic geometry of the 
Table 1 Analogies between Galois extensions and Lyashko-Looijenga extensions

Complex reflection group

Morphism:

Weights:

Extension:

Free, of rank:

Unramified

covering:

Generic fiber: $\quad \simeq W$

Ramified

part:

Discriminant:

Ramification indices:

Jacobian:$$
\operatorname{deg} v_{j}=1 ; \operatorname{deg} f_{i}=d_{i}
$$$$
|W|=d_{1} \ldots d_{n} ; \text { Galois }
$$$$
V^{\text {reg }} \rightarrow W \backslash V^{\text {reg }}
$$$$
e_{H}=\left|W_{H}\right|
$$

$$
\begin{aligned}
& p: \quad V \rightarrow W \backslash V \\
& \left(v_{1}, \ldots, v_{n}\right) \mapsto\left(f_{1}(v), \ldots, f_{n}(v)\right)
\end{aligned}
$$$$
\mathbb{C}\left[f_{1}, \ldots, f_{n}\right]=\mathbb{C}[V]^{W} \subseteq \mathbb{C}[V]
$$$$
\bigcup_{H \in \mathscr{A}} H \rightarrow(\bigcup H) / W=\mathscr{H}
$$

Lyashko-Looijenga extension

$$
\begin{aligned}
& \text { LL: } \quad Y \rightarrow \mathbb{C}^{n-1} \\
& \left(y_{1}, \ldots, y_{n-1}\right) \mapsto\left(a_{2}(y), \ldots, a_{n}(y)\right) \\
& \operatorname{deg} y_{j}=d_{j} ; \operatorname{deg} a_{i}=i h \\
& \mathbb{C}\left[a_{2}, \ldots, a_{n}\right] \subseteq \mathbb{C}\left[y_{1}, \ldots, y_{n-1}\right] \\
& n ! h^{n} /|W|=\prod^{n} i h / \prod d_{j} ; \text { non-Galois } \\
& Y-\mathscr{K} \rightarrow E_{n}^{\text {reg }}
\end{aligned}
$$

$\simeq \operatorname{Red}_{R}(c)$

$\mathscr{K}=\bigcup_{\Lambda \in \overline{\mathscr{L}_{2}}} \varphi(\Lambda) \rightarrow E_{\alpha}$

$D_{\mathrm{LL}}=\prod_{\Lambda \in \overline{\mathscr{L}}_{2}} D_{\Lambda}^{r_{\Lambda}} \in \mathbb{C}\left[a_{2}, \ldots, a_{n}\right]$

$r_{\Lambda}=$ order of parabolic elements of type $\Lambda$

$$
\Delta_{W}=\prod_{H \in \mathscr{A}} \alpha_{H}^{e_{H}} \in \mathbb{C}\left[f_{1}, \ldots, f_{n}\right]
$$

$J_{\mathrm{LL}}=\prod D_{\Lambda}^{r_{\Lambda}-1} \in \mathbb{C}\left[f_{1}, \ldots, f_{n-1}\right]$

quotient $W \backslash V$. His proof was case-by-case and detailed in an unpublished extended version of the paper [22]..$^{3}$

\subsection{The Lyashko-Looijenga extension as a virtual reflection group}

In [19] we discussed some properties of well-ramified extensions and explained that they can be regarded as an analogous of the invariant theory of reflection groups. Indeed, considering a finite graded polynomial extension $A \subseteq B$, if the polynomial algebra $A$ is the invariant algebra $B^{W}$ of $B$ under a group action, then $W$ is a complex reflection group (by Chevalley-Shephard-Todd's theorem). Here, for LL extensions, the situation is similar, but $A$ is not the invariant ring of $B$ under some group action. Still, many properties remain valid. Following Bessis, we use the term virtual reflection group for this kind of extensions. The general situation is discussed in [19].

In Table 1 we list the first analogies between the setting of a Galois extension (polynomial extension with a reflection group acting) and that of a LyashkoLooijenga extension regarded as a virtual reflection group. This is not an exhaustive list, and we may wonder if the analogies can be made further.

\section{Combinatorics of the submaximal factorizations}

In this section we are going to use properties of the morphism LL to count specific factorizations of a Coxeter element; this will lead, thanks to Theorem 4.5, to a geometric proof of a particular instantiation of Chapoton's formula.

\footnotetext{
${ }^{3}$ K. Saito, personal communication, August 2009.
} 
We call submaximal factorization of a Coxeter element $c$ a block factorization of $c$ with $(n-1)$ factors, according to Definition 1.1. Thus, submaximal factorizations contain $(n-2)$ reflections and one factor of length 2 , and are a natural first generalization of the set of reduced decompositions $\operatorname{Red}_{R}(c)$. These are included in the more general "primitive" factorizations studied in [21].

\subsection{Submaximal factorizations of type $\Lambda$}

Let $\Lambda$ be a stratum of $\overline{\mathscr{L}}$ : it corresponds (cf. Proposition 3.4) to a conjugacy class of parabolic Coxeter elements of length 2 . We say that a submaximal factorization is of type $\Lambda$ if its factor of length 2 lies in this conjugacy class. We denote by $\operatorname{FACT}_{n-1}^{\Lambda}(c)$ the set of such factorizations. Using the relations between LL and facto, we can count these factorizations.

For $\Lambda$ a stratum of $\overline{\mathscr{L}_{2}}$, let us define the following restriction of LL:

$$
\mathrm{LL}_{\Lambda}: \varphi(\Lambda) \rightarrow E_{\alpha},
$$

where $E_{\alpha}=E_{n}-E_{n}^{\text {reg }}$. We denote by $E_{\alpha}^{0}$ the subset of $E_{\alpha}$ constituted by the configurations whose partition (of multiplicities) is exactly $\alpha=2^{1} 1^{n-2}$.

We define $\varphi(\Lambda)^{0}=\mathrm{LL}_{\Lambda}^{-1}\left(E_{\alpha}^{0}\right)$, and $\mathscr{K}^{0}=\mathrm{LL}^{-1}\left(E_{\alpha}^{0}\right)=\bigcup_{\Lambda \in \mathscr{L}_{2}} \varphi(\Lambda)^{0}$. We recall from [21] the following properties:

- the restriction of LL : $\mathscr{K}^{0} \rightarrow E_{\alpha}^{0}$ is a (possibly not connected) unramified covering [21, Theorem 5.2];

- the connected components of $\mathscr{K}^{0}$ are the $\varphi(\Lambda)^{0}$ for $\Lambda \in \overline{\mathscr{L}_{2}}$;

- the image, by the map facto, of $\varphi(\Lambda)^{0}$ is exactly FACT $_{n-1}^{\Lambda}(c)$;

The map $\mathrm{LL}_{\Lambda}$ defined above is an algebraic morphism, corresponding to the extension

$$
\mathbb{C}\left[a_{2}, \ldots, a_{n}\right] /(D) \subseteq \mathbb{C}\left[f_{1}, \ldots, f_{n-1}\right] /\left(D_{\Lambda}\right)
$$

Theorem 5.1 Let $\Lambda$ be a strata of $\overline{\mathscr{L}_{2}}$. Then:

(a) $\mathrm{LL}_{\Lambda}$ is a finite quasi-homogeneous morphism of degree $\frac{(n-2) ! h^{n-1}}{|W|} \operatorname{deg} D_{\Lambda}$;

(b) the number of submaximal factorizations of $c$ of type $\Lambda$ is equal to

$$
\left|\operatorname{FACT}_{n-1}^{\Lambda}(c)\right|=\frac{(n-1) ! h^{n-1}}{|W|} \operatorname{deg} D_{\Lambda} .
$$

Proof From Hilbert series, we get that $\mathrm{LL}_{\Lambda}$ is a finite free extension of degree

$$
\frac{\prod \operatorname{deg}\left(a_{i}\right)}{\operatorname{deg}(D)} / \frac{\prod \operatorname{deg}\left(f_{i}\right)}{\operatorname{deg}\left(D_{\Lambda}\right)}=\frac{n ! h^{n}}{|W|} \frac{\operatorname{deg} D_{\Lambda}}{\operatorname{deg} D} .
$$

(a) The polynomial $D_{\mathrm{LL}}$ is a discriminant of type $A$ for the variables $a_{2}, \ldots, a_{n}$ of weights $2 h, \ldots, n h$, so we have deg $D_{\mathrm{LL}}=n(n-1) h$. Thus,

$$
\operatorname{deg}\left(\mathrm{LL}_{\Lambda}\right)=\frac{(n-2) ! h^{n-1}}{|W|} \operatorname{deg} D_{\Lambda} .
$$


(b) This degree is also the cardinality of a generic fiber of $\mathrm{LL}_{\Lambda}$, i.e., $\mid \mathrm{LL}^{-1}(\varepsilon) \cap$ $\varphi(\Lambda) \mid$ for $\varepsilon \in E_{\alpha}^{0}$. Consequently, from Property (P3) in Sect. 3.2, it counts the number of submaximal factorizations of type $\Lambda$, where the length 2 element has a fixed position (given by the composition of $n$ associated to $\varepsilon$ ). There are $(n-1)$ compositions of partition type $\alpha \vdash n$, so we obtain $\left|\operatorname{FACT}_{n-1}^{\Lambda}(c)\right|=(n-1) \operatorname{deg}\left(\operatorname{LL}_{\Lambda}\right)=$ $\frac{(n-1) ! h^{n-1}}{|W|} \operatorname{deg} D_{\Lambda}$.

Remark 5.2 Let us denote by $\operatorname{FACT}_{(2,1, \ldots, 1)}^{\Lambda}(c)$ the set of submaximal factorizations of type $\Lambda$ where the length 2 factor is in first position. By symmetry, formula (b) is equivalent to

$$
\left|\operatorname{FACT}_{(2,1, \ldots, 1)}^{\Lambda}(c)\right|=\frac{(n-2) ! h^{n-1}}{|W|} \operatorname{deg} D_{\Lambda} .
$$

As $\sum r_{\Lambda} \operatorname{deg} D_{\Lambda}=\operatorname{deg} D_{\mathrm{LL}}=n(n-1) h$, this implies the equality

$$
\sum_{\Lambda \in \overline{\mathscr{L}}_{2}} r_{\Lambda}\left|\operatorname{FACT}_{(2,1, \ldots, 1)}^{\Lambda}(c)\right|=\frac{(n-2) ! h^{n-1}}{|W|} \operatorname{deg} D_{\mathrm{LL}}=\frac{n ! h^{n}}{|W|}=\left|\operatorname{Red}_{R}(c)\right| .
$$

This formula reflects a property of the following concatenation map:

$$
\begin{gathered}
\operatorname{Red}_{R}(c) \quad \rightarrow \operatorname{FACT}_{(2,1, \ldots, 1)}(c) \\
\left(s_{1}, s_{2}, s_{3}, \ldots, s_{n}\right) \mapsto\left(s_{1} s_{2}, s_{3}, \ldots, s_{n}\right),
\end{gathered}
$$

namely, that the fiber of a factorization of type $\Lambda$ has cardinality $r_{\Lambda}$ (which is the number of factorizations of the first factor in two reflections).

Remark 5.3 In [12], motivated by the enumerative theory of the generalized noncrossing partitions, Krattenthaler and Müller defined and computed the decomposition numbers of a Coxeter element for all irreducible real reflection groups. In our terminology, these are the numbers of block factorizations according to the Coxeter type of the factors. Note that the Coxeter type of a parabolic Coxeter element is the type of its associated parabolic subgroup, in the sense of the classification of finite Coxeter groups. So the conjugacy class for a parabolic elements is a finer characteristic than the Coxeter type: take, for example, $D_{4}$, where there are three conjugacy classes of parabolic elements of type $A_{1} \times A_{1}$.

Nevertheless, when $W$ is real, most of the results obtained from formula (b) in Theorem 5.1 are very specific cases of the computations in [12]. But the method of proof is completely different, geometric instead of combinatorial. ${ }^{4}$ Note that another possible way to tackle this problem is to use a recursion, to obtain data for the group from the data for its parabolic subgroups. A recursion formula (for factorizations where the rank of each factor is dictated) is indeed given by Reading [17], but the proof is very specific to the real case.

\footnotetext{
${ }^{4}$ The computation of all decomposition numbers for complex groups, by combinatorial means, is also a work in progress (Krattenthaler, personal communication).
} 
For nonreal $W$, formula (b) implies new combinatorial results on the factorization of a Coxeter element. The numerical data for all irreducible well-generated complex reflection groups are listed in Sect. 6. In particular, we obtain (geometrically) general formulas for the submaximal factorizations of a given type in $G(e, e, n)$.

\subsection{Enumeration of submaximal factorizations of a Coxeter element}

Thanks to Theorems 4.5 and 5.1, we can now obtain a formula for the number of submaximal factorizations, with a geometric proof:

Corollary 5.4 Let $W$ be an irreducible, well-generated complex reflection group, and $d_{1} \leq \ldots \leq d_{n}=h$ be its invariant degrees. Then, the number of submaximal factorizations of a Coxeter element $c$ is equal to

$$
\left|\mathrm{FACT}_{n-1}(c)\right|=\frac{(n-1) ! h^{n-1}}{|W|}\left(\frac{(n-1)(n-2)}{2} h+\sum_{i=1}^{n-1} d_{i}\right) .
$$

Proof Using Theorem 5.1(b) and Theorem 4.5(b)-(c), we compute:

$$
\begin{aligned}
& \left|\operatorname{FACT}_{n-1}(c)\right|=\left|\operatorname{FACT}_{\alpha}(c)\right|=\sum_{\Lambda \in \overline{\mathscr{L}}_{2}}\left|\operatorname{FACT}_{n-1}^{\Lambda}(c)\right| \\
& =\frac{(n-1) ! h^{n-1}}{|W|} \sum_{\Lambda \in \overline{\mathscr{L}}_{2}} \operatorname{deg} D_{\Lambda} \\
& =\frac{(n-1) ! h^{n-1}}{|W|}\left(\operatorname{deg} D_{\mathrm{LL}}-\operatorname{deg} J_{\mathrm{LL}}\right) .
\end{aligned}
$$

As deg $D_{\mathrm{LL}}=n(n-1) h$ and $\operatorname{deg} J_{\mathrm{LL}}=\sum_{i=2}^{n} \operatorname{deg}\left(a_{i}\right)-\sum_{j=1}^{n-1} \operatorname{deg}\left(f_{j}\right)=\sum_{i=2}^{n} i h-$ $\sum_{j=1}^{n-1} d_{j}$, a quick computation gives the conclusion.

Remark 5.5 The formula in the above theorem is actually included in Chapoton's formula: indeed, there exist easy combinatorial tricks allowing us to pass from the numbers of multichains to the numbers of strict chains (which are roughly the numbers of block factorizations). We refer to [20, Appendix B] for details of these relations and general formulas for the number of block factorizations predicted by Chapoton's formula.

However, the proof we obtained here is more satisfactory (and more enlightening) than the one using Chapoton's formula. Indeed, if we sum up the ingredients of the proof, we only made use of the formula for the Lyashko-Looijenga number $n ! h^{n} /|W|$-necessary to prove the first properties of LL in [4] - the remaining being the geometric properties of LL, for which we never used the classification. In other words, we travelled from the numerology of $\operatorname{Red}_{R}(c)$ to that of $\operatorname{FACT}_{n-1}(c)$, without adding any case-by-case analysis to the setting of [4].

Although it seems to be a new interesting avenue toward a geometric explanation of Chapoton's formula, the method used here to compute the number of submaximal 
factorizations is not directly generalizable to factorizations with fewer blocks. A more promising approach would be to avoid computing explicitly these factorizations and to try to understand globally Chapoton's formula as some ramification formula for the morphism LL. A reformulation of the formula gives indeed:

$$
\forall p \in \mathbb{N}, \sum_{k=1}^{n}\left(\begin{array}{c}
p+1 \\
k
\end{array}\right)\left|\operatorname{FACT}_{k}(c)\right|=\prod_{i=1}^{n} \frac{d_{i}+p h}{d_{i}},
$$

where the $\mathrm{FACT}_{k}$ are closely related to the cardinalities of the fibers of LL.

\section{Numerical data for the factorizations of the Lyashko-Looijenga discriminants}

Here we detail explicit numerical data regarding the factorization of the discriminant polynomial $D_{\text {LL }}$.

Let us write (as in (**) in Sect. 4.2)

$$
D_{\mathrm{LL}}=\prod_{i=1}^{r} D_{i}^{p_{i}}
$$

for the factorization of $D_{\mathrm{LL}}$ into irreducible polynomials of $\mathbb{C}\left[f_{1}, \ldots, f_{n-1}\right]$.

In Table 2, we give, for each irreducible well-generated group, the weighted degrees $\operatorname{deg}\left(D_{i}\right)$ and the powers $p_{i}$ which appear in the factorization above. It is enough to deal with the 2-reflection groups, because any irreducible complex reflection group is isodiscriminantal to a 2-reflection group (see [4, Theorem 2.2]): it has the same discriminant $\Delta$ and, consequently, the same braid group and the same polynomial $D_{\mathrm{LL}}$. Thus, we only have to treat the four infinite series $A_{n}, B_{n}, I_{2}(e), G(e, e, n)$ (containing $D_{n}$ ), and 11 exceptional types (including the six exceptional Coxeter groups).

Notations In the last column of Table 2, the "LL-data":

$$
p_{1} \cdot\left(u_{1}\right)+p_{2} \cdot\left(u_{2}\right)+\ldots+p_{r} \cdot\left(u_{r}\right)
$$

means that the form of the factorization is $D_{\mathrm{LL}}=\prod_{i=1}^{r} D_{i}^{p_{i}}$ with $\operatorname{deg} D_{i}=u_{i}$. This writing reflects the additive decomposition of $\operatorname{deg} D_{\mathrm{LL}}=n(n-1) h$ (where $n=\operatorname{rk}(W)$ and $\left.h=d_{n}\right)$ in terms of the $u_{i}$ 's:

$$
\operatorname{deg} D_{\mathrm{LL}}=\sum_{i} p_{i} u_{i}
$$

By-products These numbers $\left(p_{i}, u_{i}\right)$ have many combinatorial interpretations. In particular, thanks to Theorems 4.5 and 5.1, we have:

- the number of conjugacy classes of parabolic Coxeter elements of length 2 is the number of terms in the sum (each term $\left(p_{i}, u_{i}\right)$ of the sum corresponds to one of these classes, say $\Lambda_{i}$ ); 


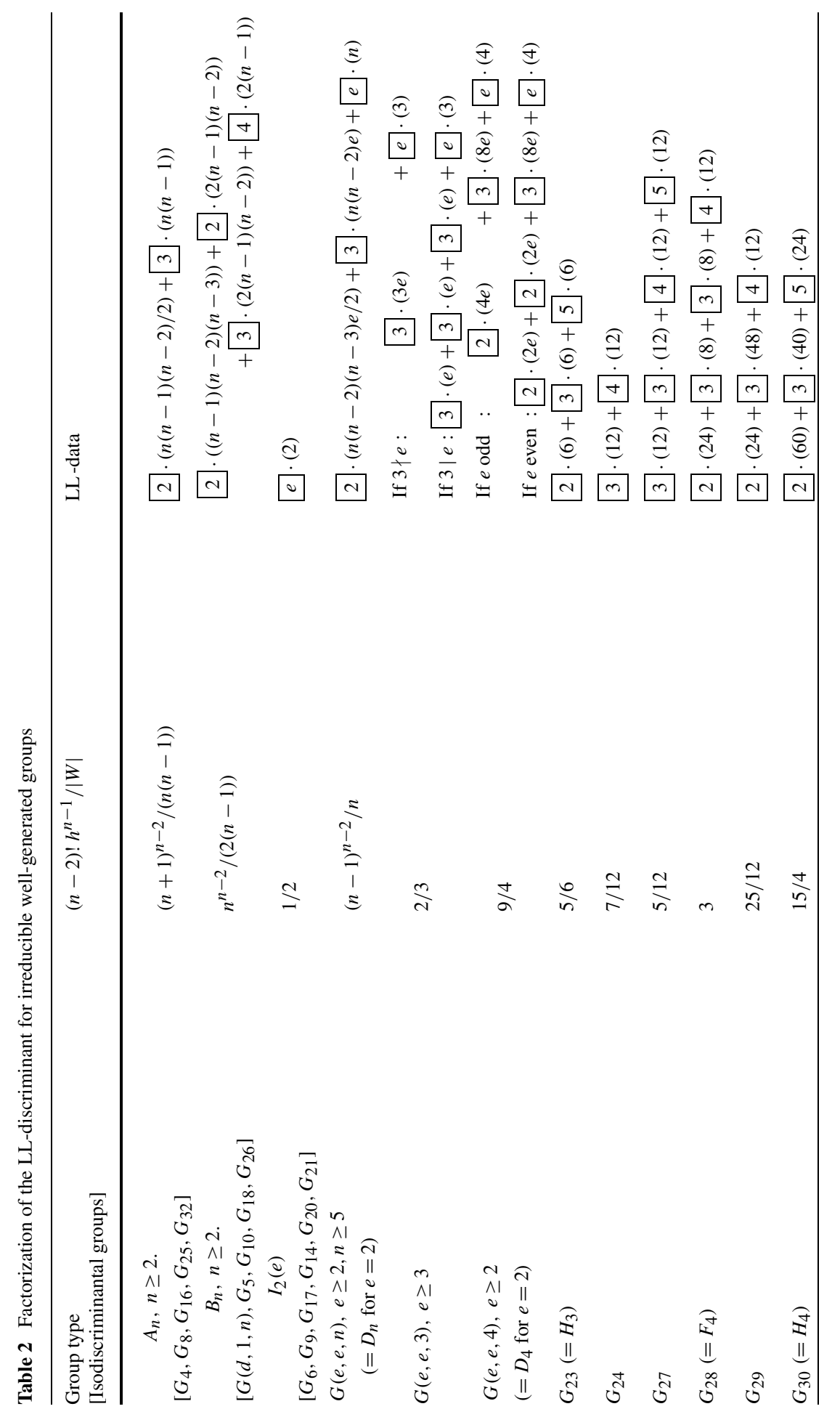




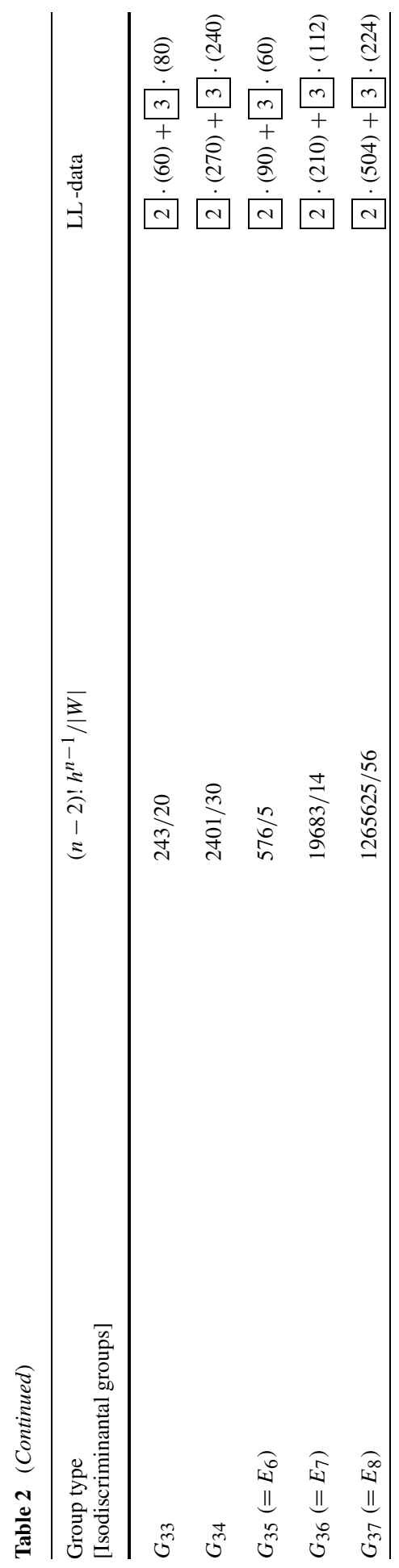


- the order of the elements in $\Lambda_{i}$ is $p_{i}$ (provided that $W$ is a 2-reflection group);

- the number $\left|\operatorname{FACT}_{(2,1, \ldots, 1)}^{\Lambda_{i}}(c)\right|$ of submaximal factorizations of a Coxeter element $c$, whose first factor is in the class $\Lambda_{i}$, equals $\frac{(n-2) ! h^{n-1}}{|W|} u_{i}$. For convenience, the first factor is also listed in the table, in the second column.

We refer to [20, Appendix A] for a detailed explanation of the computation of these data.

Acknowledgements I am grateful to Kyoji Saito for enriching mathematical exchanges during the "Hyperplane arrangements" conference in Sapporo, in August 2009. This work was part of my PhD thesis dissertation [20]. I would like to thank heartily my advisor David Bessis for his constant support and his help on many points.

\section{References}

1. Armstrong, D.: Generalized noncrossing partitions and combinatorics of Coxeter groups. Mem. Am. Math. Soc. 202(949), 159 (2009). doi:10.1090/S0065-9266-09-00565-1.

2. Athanasiadis, C.A., Reiner, V.: Noncrossing partitions for the group $D_{n}$. SIAM J. Discrete Math. 18(2), 397-417 (2004). doi:10.1137/S0895480103432192 (electronic)

3. Bessis, D.: The dual braid monoid. Ann. Sci. Éc. Norm. Super. 36(5), 647-683 (2003). doi:10.1016/j.ansens.2003.01.001

4. Bessis, D.: Finite complex reflection arrangements are $K(\pi, 1)$ (2007). http://arxiv.org/abs/math/ 0610777. Preprint arXiv:math/0610777v3

5. Bessis, D., Corran, R.: Non-crossing partitions of type (e,e,r). Adv. Math. 202(1), 1-49 (2006). doi:10.1016/j.aim.2005.03.004

6. Brady, T., Watt, C.: $K(\pi, 1)$ 's for Artin groups of finite type. In: Proceedings of the Conference on Geometric and Combinatorial Group Theory, Part I, Haifa, 2000, vol. 94, pp. 225-250 (2002). doi:10.1023/A:1020902610809

7. Chapoton, F.: Enumerative properties of generalized associahedra. Sémin. Lothar. Comb. 51, Art. B51b (2004) (electronic)

8. Deligne, P.: (1974). Letter to E. Looijenga (9/3/1974)

9. Fomin, S., Reading, N.: Generalized cluster complexes and Coxeter combinatorics. Int. Math. Res. Not. 2005(44), 2709-2757 (2005)

10. Fomin, S., Zelevinsky, A.: Cluster algebras. II. Finite type classification. Invent. Math. 154(1), 63-121 (2003). doi:10.1007/s00222-003-0302-y

11. Kane, R.: Reflection Groups and Invariant Theory. CMS Books in Mathematics/Ouvrages de Mathématiques de la SMC, vol. 5. Springer, New York (2001)

12. Krattenthaler, C., Müller, T.W.: Decomposition numbers for finite Coxeter groups and generalised non-crossing partitions. Trans. Am. Math. Soc. 362, 2732-2787 (2010)

13. Kreweras, G.: Sur les partitions non croisées d'un cycle. Discrete Math. 1(4), 333-350 (1972)

14. Lehrer, G.I., Taylor, D.E.: Unitary reflection groups. In: Australian Mathematical Society Lecture Series, vol. 20. Cambridge University Press, Cambridge (2009)

15. Looijenga, E.: The complement of the bifurcation variety of a simple singularity. Invent. Math. 23, 105-116 (1974)

16. Picantin, M.: Explicit presentations for the dual braid monoids. C. R. Math. Acad. Sci. Paris 334(10), 843-848 (2002)

17. Reading, N.: Chains in the noncrossing partition lattice. SIAM J. Discrete Math. 22(3), 875-886 (2008). doi:10.1137/07069777X

18. Reiner, V.: Non-crossing partitions for classical reflection groups. Discrete Math. 177(1-3), 195-222 (1997). doi:10.1016/S0012-365X(96)00365-2

19. Ripoll, V.: Discriminants and Jacobians of virtual reflection groups (2010). http://arxiv.org/abs/ 1001.4470. Preprint arXiv: 1001.4470

20. Ripoll, V.: Groupes de réflexion, géométrie du discriminant et partitions non-croisées. Ph.D. thesis, Université Paris Diderot-Paris 7 (2010). http://arxiv.org/abs/1010.4349. arXiv:1010.4349 
21. Ripoll, V.: Orbites d'Hurwitz des factorisations primitives d'un élément de Coxeter. J. Algebra 323(5), 1432-1453 (2010). doi:10.1016/j.jalgebra.2009.12.010

22. Saito, K.: Polyhedra dual to the Weyl chamber decomposition: a précis. Publ. Res. Inst. Math. Sci. 40(4), 1337-1384 (2004). http://projecteuclid.org/getRecord?id=euclid.prims/1145475449

23. Shephard, G.C., Todd, J.A.: Finite unitary reflection groups. Can. J. Math. 6, 274-304 (1954)

24. Springer, T.A.: Regular elements of finite reflection groups. Invent. Math. 25, 159-198 (1974)

25. Stanley, R.P.: Enumerative Combinatorics, vol. 1. Cambridge Studies in Advanced Mathematics, vol. 49. Cambridge University Press, Cambridge (1997) 